\section{Laurent Bélec Pierre Trotot Marie-Claude Lescs Françoise Gray}

\section{ADRESSES}

L. Bélec : assistant hospitalier et universitaire. Laboratoire de virologie, hôpital Broussais, Paris, France. P. Trotot : docteur en médecine, chef de service. Service de radiologie, Institut Pasteur, Paris, France. M.-C. Lescs : docteur ès sciences. Département de pathologie cellulaire et tissulaire. F. Gray : professeur des universités, praticien hospitalier. Département de pathologie cellulaire et tissulaire. Département de neurosciences médicales. Faculté de médecine de Créteil, université Paris-Val-de Marne. Laboratoirc d'anatomie pathologi que (neuropathologie), hôpital Raymond Poincaré, Garches. Département de pathologie (neuropathologic), hôpital HenriMondor, 51, avenue du Maréchal-de-Lattrede-Tassigny, 94010 Créteil, France.

$\mathrm{m} / \mathrm{s} n^{\circ} 10$ vol. 8 , décembre 92

\title{
Lésions précoces du système nerveux central au cours de l'infection par le virus de l'immunodéficience humaine
}

L'examen neuropathologique de sujets séropositifs pour le VIH, décédés aux stades précoces de la maladie, montre qu'existent des lésions du système nerveux central (SNC), probablement secondaires à une réaction immunopathologique provoquée par le virus. De semblables anomalies précoces sont également observées dans les modèles animaux d'infections par des lentivirus tels les virus de l'immunodéficience simienne (SIV) ou féline (FIV). Ces lésions de vascularite et de leptoméningite sont, normalement, spontanément régressives. Cependant, l'augmentation associée de la perméabilité de la barrière hémato-encéphalique pourrait permettre le passage dans le SNC de monocytes infectés, producteurs de virus, qui seraient ultérieurement responsables de la phase chronique de l'encéphalite associée au SIDA.

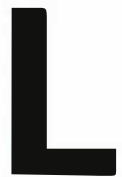

atteinte du système nerveux central (SNC) est remarquablement fréquente au cours de l'infection par le virus de l'immunodéficience humaine (VIH). Plus de $40 \%$ des cas de SIDA avérés présentent une symptomatologie neurologique patente [1] et les études neuropathologiques ont révélé l'existence de lésions du SNC dans 80 à $100 \%$ des cas. Ces complications surviennent presque toujours tard dans l'évolution de la maladie, souvent au stade terminal et du fait de l'ćvolution constamment fatale, leur aspect neuropathologique établi sur de nombreuses séries autopsiques est maintenant bien connu [2].

En revanche, bien que l'invasion pré- coce du SNC par le VIH ait été démontrée par les études virologiques, la plupart des patients ne présentent pas de troubles neurologiques pendant la période d'incubation de la maladie. L'existence de lésions précoces du SNC - évoquée sur des arguments cliniques, psychométriques, électrophysiologiques et neuroradiologiques - est discutée (Tableau I, p. 1058). Cependant, quelques études neuropathologiques, rares pour des raisons évidentes, ont montré l'existence de lésions précoces du SNC. La démonstration récente, chez l'animal, de lésions comparables, aux stades précoces d'infections par des rétro-lentivirus génétiquement proches du VIH, le simian immunodeficiency virus (SIV) et le feline immunodeficiency 


\begin{tabular}{|c|c|c|c|c|c|}
\hline \multicolumn{6}{|c|}{$\begin{array}{c}\text { ANOMALIES CLINIQUES, PARACLINIQUES ET NEUROPATHOLOGIQUES AUX STADES PRÉCOCES } \\
\text { DE L'INFECTION A VIH }\end{array}$} \\
\hline & $\begin{array}{l}\text { Manifestations } \\
\text { neurologiques } \\
\text { centrales }\end{array}$ & LCR & $\begin{array}{l}\text { Neuro- } \\
\text { imagerie }\end{array}$ & $\begin{array}{l}\text { Potentiels } \\
\text { évoqués }\end{array}$ & $\begin{array}{l}\text { Neuro-patho- } \\
\text { logie }\end{array}$ \\
\hline $\begin{array}{l}\text { Primo- } \\
\text { invasion } \\
\text { (stade } \\
\text { I/CDC) }\end{array}$ & $\begin{array}{l}\text { méningite aseptique } \\
\text { méningo- } \\
\text { encéphalite } \\
\text { myélopathie }\end{array}$ & \multicolumn{2}{|l|}{$\begin{array}{l}\text { hyperprotéinorachie } \\
\text { hypercytose à prédominance } \\
\text { lymphocytaire } \\
\text { culture du VIH + }\end{array}$} & & $\begin{array}{l}\text { leucoencé- } \\
\text { phalopathie } \\
\text { "immuno- } \\
\text { allergique" }\end{array}$ \\
\hline $\begin{array}{l}\text { Portage } \\
\text { chronique } \\
\text { (stades II } \\
\text { et III/CDC) }\end{array}$ & $\begin{array}{l}\text { méningite aseptique } \\
\text { troubles } \\
\text { neuropsychologiques, } \\
\text { discutés }\end{array}$ & $\begin{array}{l}\text { hyperprotéinorachie } \\
\text { pléiocytose } \\
\text { hyper-gamma- } \\
\text { globulinorachie et aspect } \\
\text { oligoclonal } \\
\text { culture VIH + } \\
\text { synthèse intrathécale } \\
\text { d'anticorps anti-VIH }\end{array}$ & $\begin{array}{l}\text { hyper-signaux } \\
\text { en T2 dans la } \\
\text { substance } \\
\text { blanche asy- } \\
\text { métrie de } \\
\text { distribution } \\
\text { au PET scan }\end{array}$ & $\begin{array}{l}\text { allongement de } \\
\text { latence des } \\
\text { potentiels évo- } \\
\text { qués cognitifs } \\
\text { endogènes } \\
\text { (onde P300) et } \\
\text { exogène } \\
\text { (ondes N1 et } \\
\text { P2) }\end{array}$ & $\begin{array}{l}\text { vascularite } \\
\text { cérébrale } \\
\text { méningite } \\
\text { lymphocytaire } \\
\text { pâleur myélini- } \\
\text { que proliféra- } \\
\text { tion microgliale } \\
\text { épendymite } \\
\text { granuleuse }\end{array}$ \\
\hline
\end{tabular}

RÉFÉRENCES

1. McArthur JC. Neurologic manifestations of AIDS. Medicine 1987;66 : 407-37.

2. Gray F, Geny C, Lionnet F, et al. Étude neuropathologique de 135 cas adultes de syndrome d'immunodéficience acquise (SIDA). Ann Pathol 1991; 11 : 236-47.

3. Carne CA, Tedder RS, Smith A, et al. Acute encephalopathy coincident with seroconversion for anti-HTLV-III. Lancet 1985, ii : $1206-8$

4. Ho DD, Rota TR, Schoolcy RT, et al. Isolation of HTLV-III from cerebrospinal fluid and neural tissues of patients with neurologic syndromes related to the acquired immunodeficiency syndrome. $N$ Engl J Med 1985 ; $313: 1493-7$.

5. Ho DD, Sarngadharan MG, Resnick L, Di Marzo-Veronese F, Rota TR, Hirsch MS. Primary human T-lymphotropic virus type III infection. Ann Intern Med 1985 ; $103: 880-3$

6. Denning DW, Anderson J, Rudge $\mathrm{P}$, Smith H. Acute myelopathy associated with primary infection with human immunodeficiency virus. $B r$ Med $J 1987 ; 294$ : 143-4.

7. Goudsmit J, De Wolf F, Paul DA, et al. Expression of human immunodeficiency virus antigen (HIV-Ag) in serum and cerebrospinal fluid during acute and chronic infection. Lancet 1986 ; i : 177-80.

8. Appleman ME, Marshall DW, Brey RL, et al. Cerebrospinal fluid abdnormalities in patients without AIDS who arc seropositive for the human immunodeficiency virus. virus (FIV), permet de proposer un modèle pathogénique des encéphalites spécifiques survenant au cours de ces lentiviroses.

\section{Le liquide céphalo-rachidien est précocement perturbé au cours de l'infection par le VIH}

A la phase d'invasion virale correspondant au stade I de la classification des Centers for Disease Control (CDC), le liquide céphalo-rachidien (LCR) présente des anomalies démontrant, directement ou indirectement, l'envahissement du SNC par le VIH. Une hyperprotéinorachie associée à une pléiocytose à prédominance lymphocytaire est fréquente [3-6]. Le VIH peut être isolé par culture du LCR [4,5], et des antigènes viraux ont pu y être détectés avant l'apparition des anticorps intrathécaux spécifiques [7].

Au cours du portage asymptomatique (stade II/CDC) et du syndrome de lymphadénopathies généralisées persistantes (stade III/CDC), des anomalies biologiques du LCR ont été démontrées dans la moitié des cas [8]. La barrière hémato-encéphalique étant le plus souvent normale à ces stades précoces, la majorité de ces anomalies peut être attribuée à une synthèse intrathécale [9]. Une hyper- protéinorachie modérée est fréquemment associée à une augmentation de la synthèse intrathécale des immunoglobulines, principalement des IgG, qui prennent souvent une distribution oligoclonale $[8,9]$. Il existe une synthèse intrathécale marquée d'anticorps dirigés contre les différents antigènes du VIH [10]. Cependant, l'isolement du VIH dans le LCR, qui est corrélé à la détection de l'antigène p24, est relativement difficile à ces stades et dépend de la cellularité du LCR [11]. L'hypercytose du LCR réalise une pléiocytose modéréc, avec un pourcentage élevé de monomacrophages, une diminution des lymphocytes $\mathrm{CD}^{+}$et une augmentation des lymphocytes $\mathrm{CD}^{+}{ }^{+}$, entraînant un abaissement ou une inversion du rapport $\mathrm{CD} 4{ }^{+} / \mathrm{CD}^{+}$. Ces anomalies cellulaires du LCR précèderaient celles trouvées dans le sang [12].

\section{L'atteinte précoce du SNC est le plus souvent asymptomatique}

La phase d'invasion virale est en règle cliniquement muette, cependant quelques cas d'invasion symptomatique du SNC coïncidant avec la séroconversion, et habituellement régressifs, ont été rapportés : méningite aseptique avec céphalées, fièvre, myalgies, raideur de la nuque $[4,5]$, 
encéphalopathie [3, 13] ou myélopathie [6].

Aux stades II et III du CDC, il n'existe pas de troubles neurologiques centraux manifestes. Cependant, une méningite aseptique, se traduisant par des céphalées volontiers récurrentes, est parfois signalée [1]. L'étude des fonctions neuro-psychologiques avec une batterie sophistiquée de tests psychométriques a permis à Grant $e t$ al. [14] de montrer une prévalence plus importante de troubles cognitifs chez les sujets ayant une infection à $\mathrm{VIH}$ asymptomatique. Des anomalies similaires ont été constatées chez des malades présentant un syndrome de lymphadénopathies généralisées persistantes [15]. Cependant, d'autres équipes n'ont pas trouvé que les troubles neuro-psychologiques étaient significativement plus fréquents chez les séropositifs asymptomatiques que chez les témoins $[16,17]$.

\section{Les examens paracliniques décèlent parfois des ano- malies discrètes du SNC, mais leur signification est discutée}

Les techniques d'exploration fonctionnelle du SNC et de neuro-imagerie aux stades II et III du CDC ont donné des résultats discordants.

- Bien que des anomalies aient été trouvées dans $40 \%$ des cas à l'électro-encéphalogramme [18], il semble que cet examen soit peu sensible pour détecter des anomalies du SNC aux stades précoces de l'infection à VIH.

- Un allongement des latences des potentiels évoqués auditifs et somesthésiques a été observé chez des sujets séropositifs et asymptomatiques [19]. - L'un d'entre nous (P. T.) a suivi depuis 1986, sur des examens en résonance magnétique nucléaire (IRM) cérébrale, une cohorte de 50 patients séropositifs initialement asymptomatiques. Sur le premier examen, 13 de ces patients présentaient, dans la substance blanche, de petites zones de haut signal (ZHS) d'aspect punctiforme, sur les séquences pondérées en $\mathrm{T}_{2}$ (figure $1 A$ ). Ces images n'étaient pas observées chez les témoins séronégatifs d'âge comparable. Durant cinq années de suivi longitudinal, ces lésions sont demeurées $\mathrm{m} / \mathrm{s} n^{\circ} 10$ vol. 8 , décembre 92 inchangées sur les examens semestriels successifs (figure 1B) [20]. Des résultats analogues ont été rapportés dans une autre série prospective [21] Mais d'autres auteurs [18] considèrent que les anomalies de la substance blanche observées dans leurs cohortes de sujets asymptomatiques infectés par le VIH ne sont pas significatives.

- Les études en PET scan (tomographie à émission de positons) utilisant différents traceurs [22] semblent donner des résultats plus cohérents en
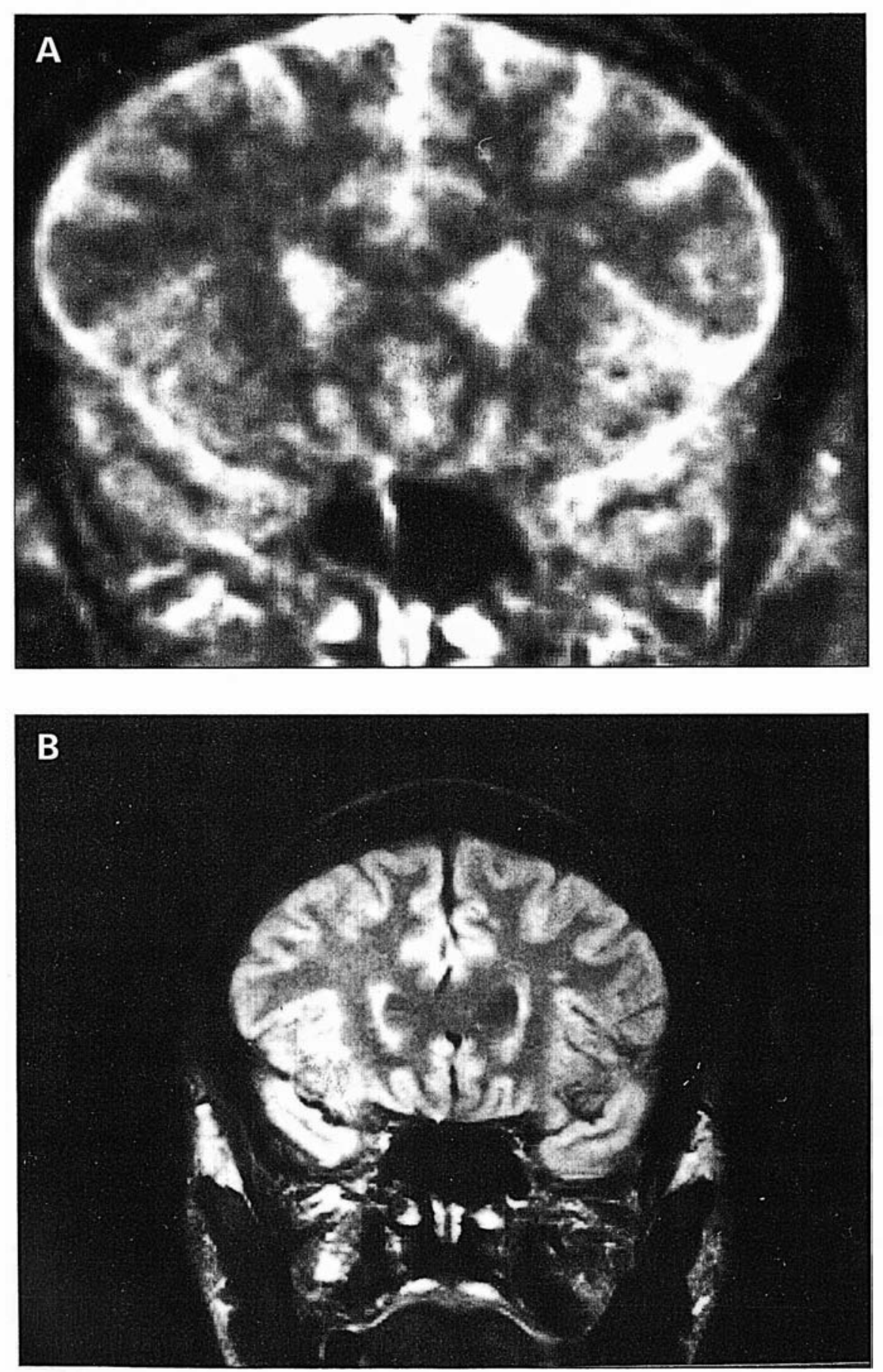

Figure 1. Zones de haut signal (ZHS) punctiformes en IRM cérébrale (séquences T2). A. Premier examen du 18 mai 1987 (0,15 Tes/a). B. Dernier examen du 30 octobre 1991 (0,15 Tesla). 
montrant une diminution de la perfusion cérébrale globale ou prédominant dans les régions frontales, chez des sujets infectés par le VIH et asymptomatiques, alors même que

\section{RÉFÉRENCES}

l'IRM ou le scanner sont normaux.

9. Elovaara I, Iivanainen $\mathrm{M}$, Valle SL, Suni J, Tervo T, Lähdevirta J. CSF protein and cellular profiles in various stages of HIV infection related to neurological manifestations $J$ Neurol Sci 1987; 78 : $331-42$.

10. Resnick L, Berger JR, Shapshak P, Tourtellottc WW. Early penctration of the blood-brain-barricr by HIV. Neurology 1988 ; $38: 9-14$.

11. Buffet R, Agut H, Chicze F, et al. Virological markers in the cerebrospinal fluid from HIV-1-infected individuals. AIDS $1991 ; 5: 1419-24$

12. Müller $\mathrm{KML}$, Elovaara I, Haltia $M$ Cercbrospinal fluid cytology in carly HIV-1 infection. In : Gray F, cd. An Atlas of the Neuropahtology of HIV Infection. Oxf ord Oxford University Press, 1993 (sous presse).

13. Nicoli F, Weill O, Dhiver C, Bille F, Gastaut JL, Gastaut JA. Manifestations ncurologiques contemporaines de la séroconversion au VIH-1. A propos d'unc obscrvation de méningo-encéphalite aiguë. Rétrovirus $1989 ; 4: 149-53$.

14. Grant I, Atkinson HJ, Hessclink JR, et al. Evidence for carly central ncrvous system involvement in the acquired immune deficicncy syndrome (AIDS) and other human immunodeficiency (HIV) infections. Studies with ncuropsychologic testing and magnetic resonance imaging. Ann Intern Med 1987 ; $107: 828-36$.

15. Janssen RS, Saykin AJ, Kaplan JE, et al. Ncurological complications of human immunodeficiency virus infection in patients with lymphadenopathy syndrome. Ann Neurol 1988 ; 23 : 49-55.

16. McArthur JC, Cohen BA, Selnes OA, et al. Low prevalence of neurological and ncuropsychological abnormalitics in otherwisc healthy HIV-1 infected individuals : results from the multicenter AIDS cohort study. Ann Neurol 1989; 26 : 601-11.

17. Sclnes OA, Miller E, McArthur MB, et al. HIV-1 infection : no cvidence of cognitive decline during asymptomatic stages. Neurology 1990 ; 40 : 204-8.

18. Koralnik IJ, Bcaumanoir A, Häusler R et al. A controlled study of carly neurologic abnormalitics in men with asymptomatic human immunodeficiency virus infection. $N$ Engl J Med 1990 ; 323 : 864-70.

19. Somma-Mauvais H, Régis H, Gastaut JL, Gastaut JA, Farnarier G. Potenticls évoqués multimodaux dans l'infection par lc virus de l'immunodéficience humainc. Rev Neurol (Paris) $1990 ; 146: 196-204$

\section{Des lésions neuropatho- logiques du SNC ont été observées aux stades précoces de l'infection par le VIH}

Les études neuropathologiques aux stades précoces de l'infection à VIH sont rares ; elles ont été réalisécs dans des circonstances particulières, comme dans les cas exceptionncls d'encéphalopathie mortelle révélatrice de l'infection à VIH, ou chez des patients séropositifs asymptomatiques morts accidentellement.

Trois cas clinico-pathologiques de leuco-encéphalopathie fulminante ont été rapportés chez les malades à des stades trés précoce [23] et précoce [24] de l'infection à VIH. Dans les deux cas de Gray el al. [24], l'étude neuropathologique a confirmé l'existence de multiples plaques de démyélinisation ayant tous les caractères des lésions de sclérose en plaques (figures $2 A$ et 2B). Le cas de Jones et al. [23], d'évolution suraiguë, avait des lésions comparables mais plus sévères et hémorragiques. Dans ces trois cas, la recherche des antigènes du VIH par immunohistochimie a été négative. La signification de ces leucoencéphalopathies précoces au cours de l'infection à VIH n'est pas connuc, mais une rclation étiopathogénique entre l'infection à VIH et la leucoencéphalopathic est très probable [24]. Une telle relation a déjà été évoquée par Berger el al. [25], qui ont rapporté trois cas cliniques d'association simultanée de sclérose en plaques avec l'infection par le VIH. Ces observations sont à rapprocher des images en IRM décrites par Trotot el al. [20] qui sont analogues à celles que l'on peut observer au cours des poussées de sclérose en plaques (voir plus haut) (figure 1).

Des anomalies peu intenses, peu spécifiques mais comparables, compatibles avec une méningoencéphalite modérée, ont été observées par d'autres auteurs chez des sujets séropositifs morts accidentellement [26].
Gray et al. [27] ont examiné les cerveaux de 11 sujets séropositifs, décédés de mort non naturelle. Aucun ne présentait de signes viscéraux en faveur d'un SIDA avéré, ni même d'un pré-SIDA. Il existait des différences très significatives entre les cerveaux de ces patients et ceux de témoins séronégatifs, morts dans les mêmes circonstances. Une inflammation vasculairc était constamment observée chez les séropositifs. Dans six cas, elle réalisait une vascularite vraie avec infiltration de la paroi vasculaire (figure 3, p. 1062), principalement composée de lymphocytes $\mathrm{T}$ et de mono-macrophages dont la plupart exprimaient les antigènes du complexe majeur d'histocompatibilité $(\mathrm{CMH})$ de classe II. Elle était associée à une méningite (figure 4, p. 1062). Ces lésions de vascularite étaient similaires, quant à leur distribution et à leur morphologie, à celles qui ont été observées dans lc nerf et dans le muscle de malades séropositifs aux stades précoces de la maladie [28]. Les lésions de la substance blanche profonde, pâleur myélinique (figures $5 A$ el $5 B, p .1063$ ) et gliose astrocytaire, étaient significativement plus intenses et plus fréquentes chez les sujets séropositifs, et pouvaient, au moins en partie, être en rapport avec une rupture de la barrière hématoencéphalique secondaire à la vascularite [27]. L'évaluation de la densité neuronale du cortex frontal n'a pas montré de différence significative entre les séropositifs et les témoins [29]. La recherche immunohistochimique des antigènes du VIH sur des coupes de tissu cérébral incluses en paraffine était négative dans tous les cas. Dans un seul cas, où l'on disposait de tissu congelé, l'amplification par polymérisation en chaîne (PCR) a montré la présence de petites quantités de génome viral [30].

L'examen neuropathologique d'une série de patients hémophiles infectés par le VIH, chez qui l'évolution de la maladie est souvent accélérée du fait de complications propres à l'hémophilie comme une hémorragie cérébrale ou une cirrhose posthépatitique, a montré qu'ils présentaient rarement des infections opportunistes ou une encéphalite à VIH qui surviennent tard dans l'évolution 

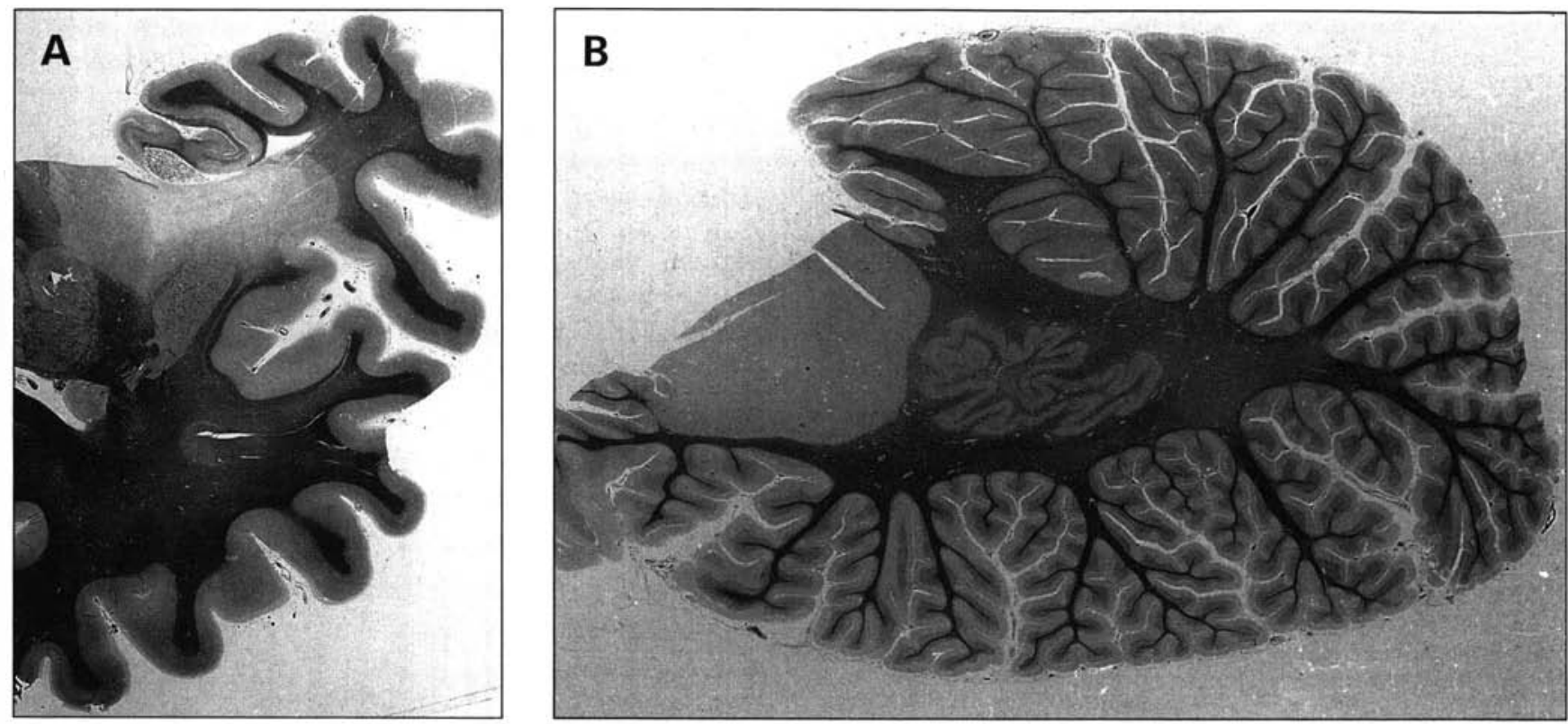

Figure 2. Plaques de démyélinisation à l'emporte-pièce ayant les caractères de la sclérose en plaques dans un cas de leucoencéphalopathie fulminante révélatrice de l'infection à VIH. A. Coupe vertico-frontale de l'hémisphère cérébral gauche passant par le noyau rouge, coloration myélinique de Loyez. B. Coupe horizontale de l'hémisphère cérébelleux gauche, coloration myélinique de Loyez.

de l'infection à VIH. En revanche, ils avaient très souvent des lésions peu spécifiques, compatibles avec une méningo-encéphalite modérée [31].

Des lésions comparables mais moins, ou non, inflammatoires ont été décrites à des stades plus avancés de la maladie. Chez un malade au stade de pré-SIDA, présentant des signes de démence débutante, on constatait une pâleur myélinique modérée avec gliose astrocytaire de la substance blanche, en l'absence de signes inflammatoires, de cellules géantes multinucléées, ou d'atrophie cérébrale [32]. Les mêmes aspects étaient aussi observés chez les patients ayant une symptomatologie démentielle modérée qui pourrait correspondre à un stade plus précoce de la maladie [33]. Dans les cas de SIDA avéré, sans troubles neurologiques ni anomalies neuroradiologiques, des lésions " non diagnostiques " - telles qu'une épendymite granuleuse, un épaississement fibreux des méninges, une pâleur myélinique avec astrocytose réactive et/ou prolifération microgliale, et une minéralisation des parois vasculaires - sont fréquentes et pourraient représenter les séquelles d'une leptoméningite et/ou d'une vascularite cérébrale [2].

\section{Certaines lentiviroses animales s'accompagnent d'encéphalopathies précoces}

L'encéphalite survenant au cours de l'infection par le Visna Maedi chez le mouton constitue le premier modèle animal d'encéphalite transmissible provoquée par un lentivirus. Le virus Visna Maedi a un tropisme prononcé pour les monocytes et les macrophages. Une réplication virale permanente a minima au sein des monomacrophages infectés présents dans le tissu cérébral pourrait être à l'origine d'une encéphalopathie progressive, plus par activation de cellules immunitaires dirigées contre certains antigènes viraux, que par l'effet cytopathogène du virus lui-même. Les lésions cérébrales, inflammatoires et démyélinisantes, sont assez voisines de celles des leuco-encéphalopathies fulminantes révélatrices de l'infection à VIH (voir plus haut) qui pourraient relever d'un mécanisme analogue [34].

L'infection par le FIV (virus de l'immunodéficience féline) comporte trois phases : (1) l'infection primaire qui associe une virémie de plusieurs semaines à un syndrome de lympha- dénopathie généralisée, une neutropénie et une lymphopénie ; (2) une phase asymptomatique durant plusieurs années ; (3) une phase terminale caractérisée par une immunodépression au cours de laquelle peut survenir une encéphalopathie spécifique. Chez le chat expérimentalement infecté par le FIV, des lésions du SNC comparables à celles observées chez l'homme sont présentes deux mois après l'inoculation et persistent, identiques, jusqu'à 12 mois, sans signes d'encéphalite spécifique. Chez le chat naturellement infecté, des lésions neuropathologiques similaires sont constamment trouvées avec, très rarement, des cellules géantes multinucléées [35].

L'infection par le SIV (virus de l'immunodéficience simienne) présente de nombreuses analogies avec l'infection par le VIH, puisqu'elle induit avec la même fréquence, chez le macaque, une immunodéficience et une encéphalite spécifique. Chez des macaques inoculés par voie intraveineuse, jusqu'à un mois après l'injection, il existait des lésions cérébrales superposables à celles décrites chez l'homme; en outre, les antigènes du $\mathrm{CMH}$ de classe II étaient fortement exprimés par les cellules 


\section{RÉFÉRENCES}

20. Trotot PM, Sansonetti PJ, Levillain R, Cabanis EA, Lavayssière R, Sandoz-Tronca C. Imagerie par résonance magnétique : dépistage précoce des atteintes du système nerveux central au cours du syndrome d'immunodéficicence acquise (SIDA). $C R$ Acad Sci Paris 1988; 307 : 1-4.

21. Donovan Post MJ, Levin BE, Berger $J R$, Duncan R, Quencer RM, Calabro G. Sequential cranial MR findings of asymptomatic and neurologically symptomatic HIV + subjects. AJNR $1992 ; 13: 359-70$.

22. Pascal S, Resnick L, Barker WW, et al. Metabolic asymmetrics in asymptomatic HIV-1 seropositive subjects : relationship to disease onset and MRI findings. $J \mathrm{Nucl} \mathrm{Med}$ $1991 ; 32$ : 1725-9.

23. Jones HR Jr, Ho DD, Forgacs $P$, et al. Acute fulminating fatal leukoencephalopathy as the only manifestation of human immunodeficiency virus infection. Ann Neurol $1988 ; 23$ : 519-22.

24. Gray F, Chimelli L, Mohr M, Clavelou P, Scaravilli F, Poirier J. Fulminating multiple sclerosis-like leukoencephalopathy revealing human immunodeficiency virus infection. Neurology 1991； 41: 105-9.

25. Berger JR, Sheremata WA, Resnick L, Atherton S, Fletcher MA, Norenberg $M$. Multiple sclerosis-like illness occurring with human immunodeficiency virus infection. Neurology $1989 ; 39$ : 324-9.

26. Lenhardt TM, Super MA, Wiley CA. Neuropathological changes in an asymptomatic HIV seropositive man. Ann Neurol $1988 ; 23: 209-10$

27. Gray F, Lescs MC, Keohane C, et al. Early brain changes in HIV infection : neuropathological study of 11 HIV seropositive, non-AIDS cases. J Neuropathol Exp Neurol $1992 ; 51: 177-85$

28. Gherardi RK, Mhiri C, Baudrimont $\mathrm{M}$, et al. Iron pigment deposits, small vessel vasculitis, and erythrophagocytosis in the muscle of human immunodeficiency virusinfected patients. Hum Pathol 1991; 22 : 1187-94.

29. Everall IP, Gray F, Barnes H, Durigon $M$, Luther P, Lantos P. Neuronal loss does not occur in asymptomatic HIV infec- microgliales, témoignant d'un possible mécanisme immunopathologique [36]. Le SIV pouvait être détecté par hybridation in situ dans le tissu nerveux dès le $7 e$ jour après l'inoculation dans des cellules d'origine mono-macrophagique; ce qui laisse penser que des mono-macrophages infectés traversent précocement la barrière hémato-encéphalique. Une véritable encéphalite à SIV avec des cellules géantes multinucléées ne pouvait être diagnostiquée que deux mois ou plus après l'inoculation. Aux stades précoces, contrairement à ce que l'on observe aux stades tardifs, les cellules infectées par le SIV étaient peu nombreuses et exprimaient une faible quantité d'ARN viral, suggérant l'existence d'un contrôle immunologique de la réplication virale dans le SNC, pendant la période de latence.

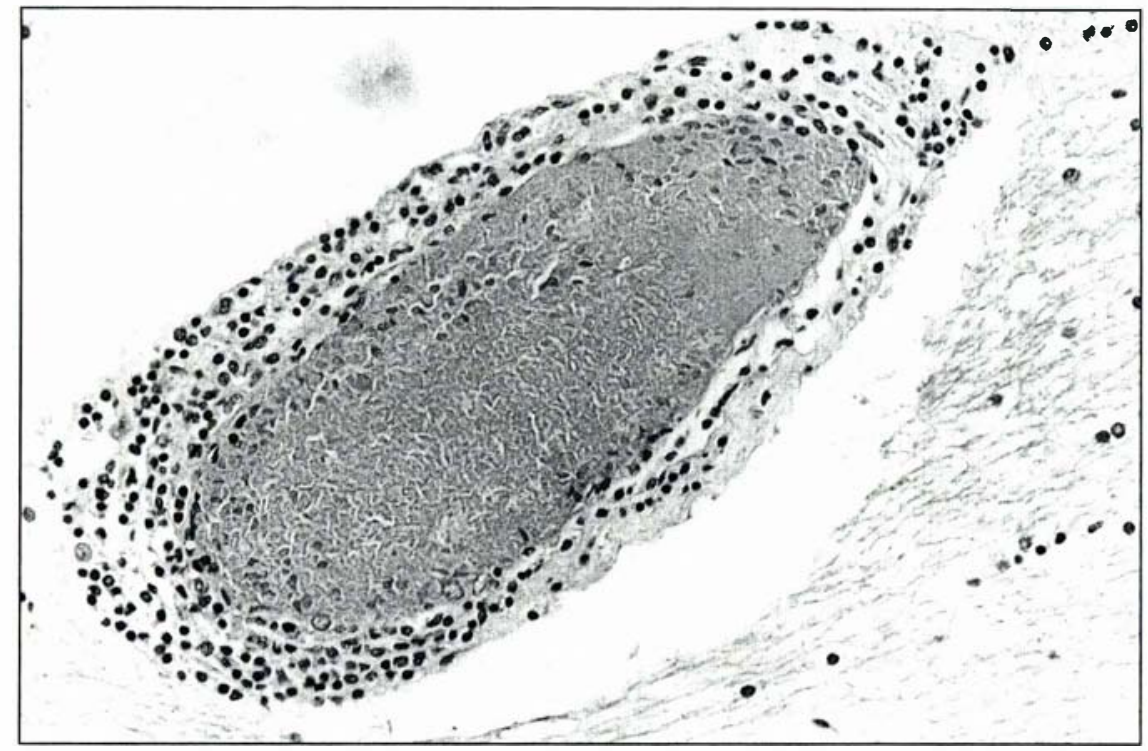

Figure 3. Inflammation périvasculaire. Infiltration de la paroi d'une veinule de la susbtance blanche par des cellules mononucléées (hématoxyline/éosine, $\times 250)$.

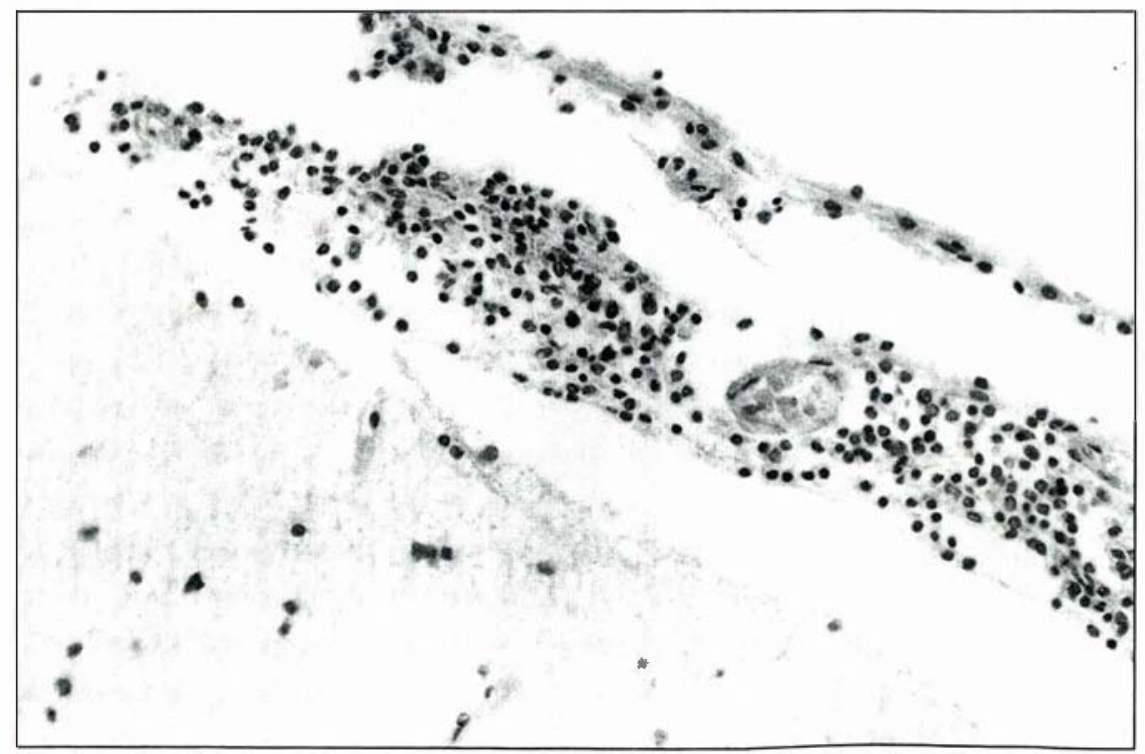

Figure 4. Inflammation des leptoméninges. Infiltration des espaces leptoméningés par des cellules inflammatoires mononucléées (hématoxyline/éosine $\times$ 250). 
Quelles hypothèses peuton avancer concernant la pathogénie des lésions précoces du SNC au cours de l'infection par le VIH ?

La pathogénie des lésions précoces du SNC au cours de l'infection par le VIH demeure spéculative. Bien que l'ADN viral puisse être détecté, en petite quantité, dans le SNC aux stades précoces de l'infection par le VIH [30] et par le SIV [36], il est peu probable que les lésions observées soient imputables à la multiplication virale elle-même. En effet, la présence de cellules géantes multinucléées témoignant d'une infection productive à VIH n'a été constatée dans aucun cas $[23,24,26,27,32$, 33 ], et les recherches immunohistologiques d'antigènes du VIH dans le tissu cérébral sont restées négatives [23, 24, 27]. Un processus de nature immunopathologique, contemporain de la primo-infection pour le VIH, est plus probablement en cause comme le suggèrent l'existence d'encéphalopathies démyélinisantes révélatrices de l'infection à VIH [23-25] et les quelques observations de syndrome de Guillain Barré coïncidant avec la séroconversion pour le VIH [37]. Il pourrait expliquer l'afflux de mono-macrophages infectés dans le SNC. La vascularite observée à cette occasion pourrait favoriser la pénétration du virus dans le SNC, probablement par l'intermédiaire d'un mono-macrophage infecté, franchissant la barrière hématoencéphalique, et jouant le rôle du "cheval de Troie" [38].

Figure 5. Pâleur myélinique de la substance blanche profonde chez un héroïnomane séropositif pour le VIH, asymptomatique, mort d'overdose opiacée. A. Coupe verticofrontale de l'hémisphère cérébral gauche passant par le splénium du corps calleux, coloration myélinique de Loyez. B. Coupe horizontale du cervelet et du tronc cérébral passant par la protubérance basse, coloration myélinique de Loyez.

$\mathrm{m} / \mathrm{s} n^{\circ} 10 \mathrm{vol} .8$, dicembre 92
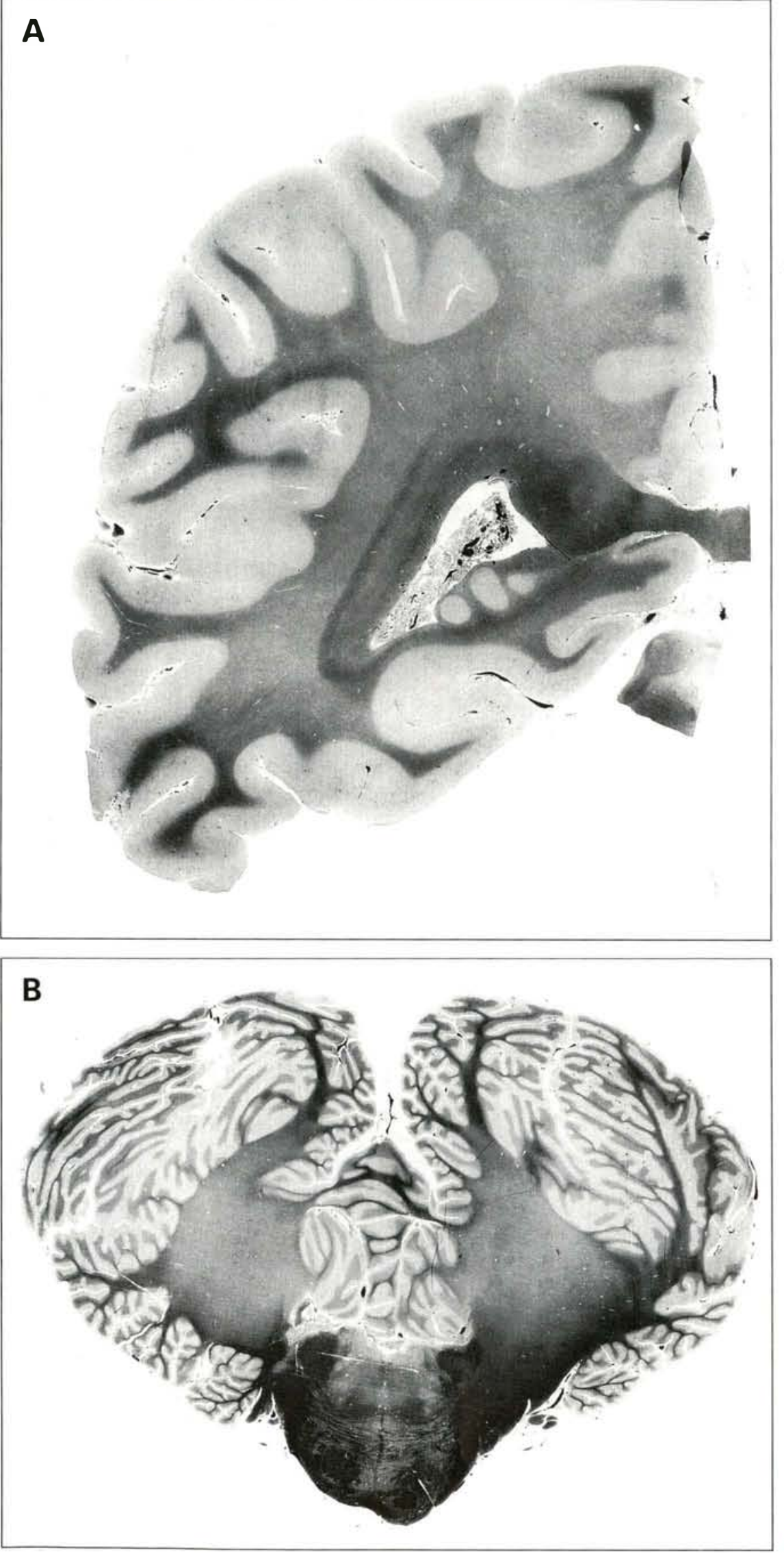


\section{RÉFÉRENCES}

30. Sinclair E, Gray F, Scaravilli F. PCR detection of HIV proviral DNA in the brain of an asymptomatic HIV-positive patient. $J$ Neurol 1992 ; 239 : 469-71.

31. Esiri MM, Scaravilli F, Millard PR, Harcourt-Webster JN. Neuropathology of HIV infection in haemophiliacs : comparative necropsy study. $B r$ Med J $1989 ; 299$ : 1312-6.

32. McArthur JC, Becker PS, Parisi JE, et al. Neuropathological changes in early HIV-1 dementia. Ann Neurol $1989 ; 26$ : $681-4$.

33. Price RW, Sidtis JJ, Brew BJ. AIDS dementia complex and HIV-1 infection : a view from the clinic. Brain Pathol 1991; 1 : 155-62.

34. Georgson G, Pálsson PA, Pétursson G Some comparative aspects of VISNA and AIDS. In : Racz P, Haase AT, Gluckman JC, eds. Modern pathology of AIDS and other retroviral infections. Basel : Karger, 1990 : 82-98.

35. Hurtrel M, Ganière JP, Guelfi JF, $e$ al. Comparison of early and late feline immunodeficiency virus encephalopathies. AIDS 1992 ; 6 : 399-406.

36. Chakrabarti L, Hurtrel M, Maire MA, et al. Early viral replication in the brain o SIV-infected Rhesus monkeys. Am J Pathol $1991 ; 139$ : 1273-80.

37. Persuy P, Arnott G, Fortier B, et al. Syndrome de Guillain et Barré d'évolution favorable dans un cas d'infection récente par le virus de l'immunodéficience humaine. Rev Neurol (Paris) $1988 ; 144: 32-5$.

38. Ho DD, Pomerantz RJ, Kaplan JC. Pathogenesis of infection with human immunodeficiency virus. $N$ Engl $J$ Med 1987 ; $317: 278-86$

39. Belman AL, Lantos G, Horoupian D, et al. AIDS : calcification of the basal ganglia in infants and children. Neurology 1986 36 : 1192-9.

40. Bélec L, Gray F, Mikol J, et al. Cytamegalovirus (CMV) encephalomyeloradiculitis and human immunodeficiency virus (HIV) encephalitis : presence of HIV and CMV co-infected multinucleated giant cells
Ce processus immunopathologique serait habituellement transitoire et réversible, les lésions neuropathologiques observées au stade d'incubation représentant vraisemblablement des cicatrices de méningite ou de vascularite cérébrale précoces. Cette interprétation est en accord avec l'hypothèse que la minéralisation des parois vasculaires fréquemment trouvée au cours du SIDA serait secondaire à une vascularite avec dépôts de complexes immuns, et avec les études neuroradiologiques montrant qu'il existe, chez les enfants sidéens, une rupture de la barrière hématoencéphalique avec odème et inflammation, précédant la calcification des noyaux gris centraux [39]. Pendant la période d'incubation, le VIH ne peut être détecté qu'occasionnellement dans les macrophages ou dans la microglie périvasculaire, qui constituent un réservoir pour le virus, suggérant que la multiplication virale est contrôlée par le système immunitaire. Contrairement aux stades tardifs de la maladie, aucune perte neuronale ne peut être détectée chez les sujets asymptomatiques [29]. Cela est en accord avec l'hypothèse que la perte neuronale est liée à l'intensité de la réplication virale.

Ultérieurement, la survenue d'une encéphalite à VIH dépendrait, au moins en partie, de l'aggravation de l'immunosuppression cellulaire. La réplication du VIH au sein des monocytes pourrait être activée par différents cofacteurs comme les infections opportunistes. Cette activation pourrait résulter de la simple transformation en macrophages, des monocytes attirés dans le foyer inflammatoire. Un mécanisme plus spécifique d'activation de la transcription du VIH pourrait aussi être lié à une interaction bidirectionnelle entre le VIH et certains virus du groupe herpès [40]

\section{Summary}

Early brain changes in human immunodeficiency virus (HIV)infection

Early HIV infection of the central nervous system has been demonstrated by cerebrospinal fluid studies. However, most HIV infected individuals remain neurologically unimpaired during the incubation period. A few psychometric, radiological, and electrophysiological studies suggest that neurological abnormalities are present at the initial stages of HIV infection, but their results are controversial and, for obvious reasons, there are few supportive neuropathological data. Examination of the brains of HIVpositive asymptomatic individuals who died from unnatural causes, and of rare cases with acute, fatal encephalopathy revealing HIVinfection, and comparison with early experimental simian immunodeficiency virus and feline immunodeficiency encephalopathy demonstrated the presence of early brain changes, including leptomeningitis and vasculitis with myelin pallor and gliosis of the deep white matter, likely to be secondary to vascular inflammation through an opening of the blood brain barrier. Although small amounts of HIV genome could be detected by PCR in a few cases, these changes do not seem to result from a productive HIV infection in the CNS, as seen in HIV encephalitis. The occurrence of a usually asymptomatic and transient immunopathologic reaction coinciding with early HIV infection of the nervous system appears to be more likely.

TIRÉS A PART

F. Gray. 